\title{
ON ASYMPTOTIC FORMULA FOR ELECTRIC RESISTANCE OF CONDUCTOR WITH SMALL CONTACTS
}

\author{
R.R. GADYL'SHIN, A.A. ERSHOV, S.V. REPYEVSKY
}

\begin{abstract}
We construct and justify rigorously the complete asymptotic expansion for the electric resistance of a three-dimensional resistance connected by two small contacts of arbitrary shape. We obtain explicit formulae for the first two terms in the asymptotics generalizing the classical Holm formula of one-term asymptotics for two small round contacts of same radius.
\end{abstract}

Keywords: electrical resistance, small contacts, Holm formula, asymptotic expansion, boundary value problem, the method of matching asymptotic expansions, Laplace equation, mixed problem.

Mathematics Subject Classification: 35J05, 35J25, 35B40, 78M35

\section{INTRODUCTION}

Work [1] is one of the first papers where the well-known Holm approximation of electric resistance of an arbitrarily shaped pattern was mentioned:

$$
R(\varepsilon)=\frac{1}{2 \varepsilon \sigma}+O(1), \quad \varepsilon \rightarrow 0
$$

where $\varepsilon$ is the radius of small round contacts on a flat part of a surface, $\sigma$ is the conductivity of a material. In the experimental test made in [2] the error of formula (11) did not exceed $\pm 1,5 \%$. In the monograph by R. Holm [3] an analytic justification of formula (1) was provided. Holm approximation (11) is often employed in the modern problems, see, for instance, [4], [5].

In the present work we consider the case of arbitrarily shaped contacts not necessarily being the same. We construct the complete asymptotics for the resistance. For the leading term of the asymptotics we obtain an explicit formula, in which we reflect the dependence on the geometry of the contacts, cf. formulae (2), (3) below. We also provide the formula for the next-to-leading term in the asymptotics for the resistance.

In order to do it, in the present work we construct the complete asymptotic expansion for the solution of the boundary value problem describing the electric potential of the conductor connected by means of small or so-called "point" contacts (see, for instance, [6]). By this expansion we find the asymptotic for the electric resistance.

In conclusion we mention that a two-dimensional analogue of similar studies was provided in works [7-9].

R.R. Gadyl'shin, A.A. Ershov, S.V. Repyevsky, On asymptotic FORMUla FOR EleCtric ResisTANCE OF CONDUCTOR WITH SMALL CONTACTS.

(C) Gadyl'shin R.R., Ershov A.A., Rep'evskij S.V. 2015.

The work of the first author is made in the framework of basic part of state task in the field of scientific activity of Ministery of Education and Science of Russia. The work of the second author is supported by RFBR project no. 14-31-50424-mol_nr and the Foundation for supporting young scientists "Moebius contest". The third author is partially supported by RFBR project no. 14-01-00322.

Submitted April 15, 2015. 


\section{Formulation OF PROBlem AND MAin RESUlts}

Let $x=\left(x_{1}, x_{2}, x_{3}\right)$, a conductor $\Omega$ be a bounded simply-connected domain in $\mathbb{R}^{3}$ with boundary $\partial \Omega \in C^{\infty}$. We assume that this boundary has two flat parts to which we associate two Cartesian coordinate systems $O_{+} x_{1}^{+} x_{2}^{+} x_{3}^{+}$and $O_{-} x_{1}^{-} x_{2}^{-} x_{3}^{-} ; x_{ \pm}:=\left(x_{1}^{ \pm}, x_{2}^{ \pm}, x_{3}^{ \pm}\right)$. Let us describe the shape of small contacts $\gamma_{+}^{\varepsilon}$ and $\gamma_{-}^{\varepsilon}$ in these coordinates. Let sets $\gamma_{+}$and $\gamma_{-}$are the closures of bounded simply connected domains on the planes $x_{3}^{+}=0$ and $x_{3}^{-}=0$, respectively, $\partial \gamma_{ \pm} \in C^{\infty}$. As the cross-sections of the small contacts we choose $\gamma_{ \pm}^{\varepsilon}=\left\{x_{ \pm}: \varepsilon^{-1} x_{ \pm} \in \gamma_{ \pm}\right\}$, $0<\varepsilon \ll 1$ (Fig. 11).

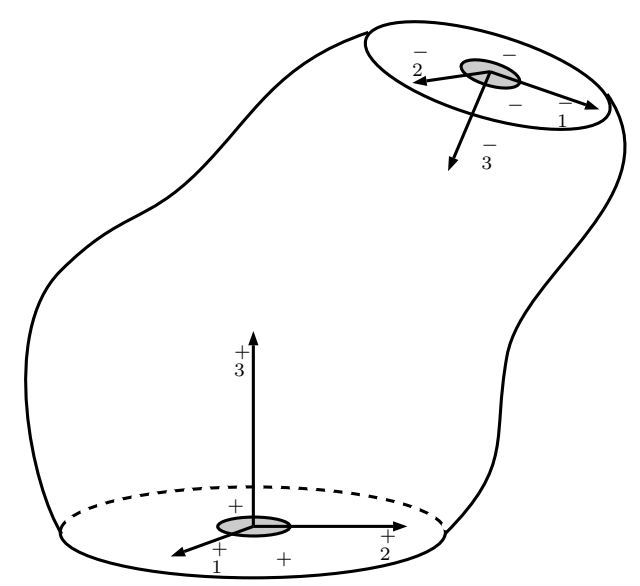

Figure 1. Conductor $\Omega$

The first coordinate system will be regarded as the main one. This is why sometimes we shall omit the symbol "+" and we shall write it as $O x_{1} x_{2} x_{3} ; x:=\left(x_{1}, x_{2}, x_{3}\right)$.

In the work we obtain the following asymptotic formula for the electric resistance:

$$
R(\varepsilon)=\frac{R_{-1}}{\varepsilon}+R_{0}+\sum_{j=1}^{\infty} \varepsilon^{j} R_{j}
$$

where

$$
R_{-1}=\frac{C_{\gamma_{+}}+C_{\gamma_{-}}}{2 \pi \sigma C_{\gamma_{+}} C_{\gamma_{-}}}, \quad R_{0}=-\frac{1}{2 \pi \sigma}\left(G_{+}\left(O_{-}\right)+G_{-}\left(O_{+}\right)\right) .
$$

Hereinafter $C_{\gamma_{ \pm}}>0$ are the capacities of disks $\gamma_{ \pm}$(see, for instance, [10, Ch. 2, Sect. 1], [11, Ch .2 , Sect. 3]), $G_{ \pm}(x)=r_{ \pm}^{-1}+g_{ \pm}(x)$, where $r_{+}=|x|, r_{-}=\left|x^{-}\right|$, while functions $g_{ \pm}(x) \in C^{\infty}(\bar{\Omega})$ solve the boundary value problems

$$
\left\{\begin{array}{l}
\Delta g_{ \pm}=0, \quad x \in \Omega \\
\frac{\partial g_{ \pm}}{\partial \mathbf{n}}=-2 \pi-\frac{\partial}{\partial \mathbf{n}}\left(\frac{1}{r_{ \pm}}\right), \quad x \in \partial \Omega \backslash O_{ \pm}, \quad g_{ \pm}\left(O_{ \pm}\right)=0
\end{array}\right.
$$

where $\mathbf{n}$ is the outward normal. The existence of such functions is well-known ( [12, Ch. 2, Sect. 2, Subsect. 122], [13, Ch. 6, Sect. 7,8]).

If $\gamma_{ \pm}$are the unit circles, then $C_{\gamma_{ \pm}}=\frac{2}{\pi}$ [11, Ch. 2, Sect .3], [14, Ch. 1, Sect. 4]. This is why even for the particular case of small circular contacts $\gamma_{ \pm}^{\varepsilon}$ of radius $\varepsilon$ considered in [1-3], by (2), (3) we obtain the identity

$$
R(\varepsilon)=\frac{1}{2 \sigma \varepsilon}-\frac{1}{2 \pi \sigma}\left(G_{-}\left(O_{+}\right)+G_{+}\left(O_{-}\right)\right)+O(\varepsilon)
$$


specifying classical formula (1). We note that for certain shapes of disks $\gamma$ their capacities $C_{\gamma}$ can be found explicitly [10,11. In particular, if $\gamma_{ \pm}$is the ellipse with axes $a$ and $b$, then

$$
C_{\gamma_{ \pm}}=\frac{a}{K\left(\sqrt{\left|a^{2}-b^{2}\right|} / a\right)}
$$

where

$$
K(z)=\int_{0}^{\pi / 2} \frac{d t}{\sqrt{1-z^{2} \sin ^{2} t}}
$$

is the complete integral of the first kind [11, Ch. 2, Sect. 3].

We also observe that the flatting of the boundary at the place of connecting of contacts is, first, natural from the technological point of view and, second, makes no influence on the leading term in the asymptotics (cf. the remark in the next section).

\section{REDUCing OF CALCULATING RESISTANCE TO SOLVING BOUNDARY VALUE PROBLEM}

Since by the Ohm's law

$$
R=\frac{\Delta U}{I}
$$

where $\Delta U$ is the difference of the potentials at the contacts, and $I$ is the current strength passing through the conductor, knowing electric potential $u(x, \varepsilon)$ at each point of conductor $\Omega$, we can calculate the difference of potentials $\Delta U=\left|u\left(O_{+}\right)-u\left(O_{-}\right)\right|$and the current strength as the absolute value of the surface integral over any cross-section $H$ of the conductor:

$$
I=\left|\sigma \int_{H} \frac{\partial u}{\partial \mathbf{n}_{H}} d S\right|,
$$

where $\mathbf{n}_{H}$ is the normal to the cross-section $H$, and $\sigma \frac{\partial u}{\partial \mathbf{n}_{H}}$ is the density of the current strength passing through the cross-section $H$ (cf., for instance, [14, Ch. 3, Sect. 21]).

Electric potential $u(x, \varepsilon)$ is modeled by means of solution to the following boundary value problem:

$$
\begin{cases}\Delta u=0, & x \in \Omega, \\ \frac{\partial u}{\partial \mathbf{n}}=0, & x \in \partial \Omega \backslash\left\{\gamma_{+}^{\varepsilon} \cup \gamma_{-}^{\varepsilon}\right\}, \\ u=U_{ \pm}, & x \in \gamma_{ \pm}^{\varepsilon},\end{cases}
$$

where $U_{+}, U_{-}$are the potentials on contact surfaces, $\mathbf{n}$ is the outward normal [14, Ch. 3, Sect. 21], [3, Sect. 4].

Since the sought resistance of conductor is independent of the voltage, for simplicity we let $U_{+}=1, U_{-}=-1$. That is, instead of (7) it is sufficient to consider the following main boundary value problem

$$
\begin{cases}\Delta u=0, & x \in \Omega, \\ \frac{\partial u}{\partial \mathbf{n}}=0, & x \in \partial \Omega \backslash\left\{\gamma_{+}^{\varepsilon} \cup \gamma_{-}^{\varepsilon}\right\}, \\ u= \pm 1, & x \in \gamma_{ \pm}^{\varepsilon} .\end{cases}
$$

By [15] and smoothness improving theorems for solutions to elliptic equations [16, Ch. IV, Sect. 2, Subsect. 3] we obtain the unique solvability of problem (88) in the class of functions $C^{\infty}\left(\bar{\Omega} \backslash\left\{\partial \gamma_{+}^{\varepsilon} \cup \partial \gamma_{-}^{\varepsilon}\right\}\right) \cap C(\bar{\Omega})$. 
Remark 1. If the boundary is not flat in the vicinity of contacts connection, it can be made flat by a local change of variables. Of course, Laplace operator becomes an operator with variable coefficients that makes no influence on calculating the leading terms of the asymptotics. At the same time, it makes the procedure of constructing the complete asymptotics longer and more complicated technically, see [17], where the eigenvalue problem for a second order elliptic operator with variable coefficients and a change of the type of boundary condition on a small piece of the boundary was considered. We observe that a similar situation appears also for other singular perturbations contracting to a point (for small holes see [18], [19]).

\section{CONSTRUCTION OF LEADING TERMS OF ASYMPtotics FOR SOLUTION TO BOUNDARY VALUE PROBLEM (8)}

We construct the formal asymptotic for solution $u(x, \varepsilon)$ to boundary value problem (8) as $\varepsilon \rightarrow 0$ by the method of matching asymptotic expansion [18], [20], [21].

Following this method, it is natural to construct the asymptotic expansion of function $u(x, \varepsilon)$ in the vicinity of the parts of the boundary $\gamma_{ \pm}^{\varepsilon}$ (i.e., in the vicinity of points $O_{ \pm}$) as

$$
u(x, \varepsilon)=v_{0}^{ \pm}\left(\frac{x_{ \pm}}{\varepsilon}\right)+\varepsilon v_{1}^{ \pm}\left(\frac{x_{ \pm}}{\varepsilon}\right)+\sum_{j=2}^{\infty} \varepsilon^{j} v_{j}^{ \pm}\left(\frac{x_{ \pm}}{\varepsilon}\right) .
$$

Substituting series (9) into (8) and passing to internal variables $\xi_{ \pm}=\frac{x_{ \pm}}{\varepsilon}$, we obtain the boundary value problems for internal expansions:

$$
\begin{aligned}
& \begin{cases}\Delta_{\xi_{ \pm}} v_{0}^{ \pm}=0, & \xi_{3}^{ \pm}>0, \\
v_{0}^{ \pm}= \pm 1, & \xi_{ \pm} \in \gamma_{ \pm}, \\
\frac{\partial v_{0}^{ \pm}}{\partial \xi_{3}}=0, & \xi_{ \pm} \in \Gamma_{ \pm}:=\left\{\xi_{ \pm}: \xi_{3}^{ \pm}=0, \xi_{ \pm} \notin \gamma_{ \pm}\right\},\end{cases} \\
& \begin{cases}\Delta_{\xi_{ \pm}} v_{j}^{ \pm}=0, & \xi_{3}^{ \pm}>0, \\
v_{j}^{ \pm}=0, & \xi_{ \pm} \in \gamma_{ \pm}, \\
\frac{\partial v_{j}^{ \pm}}{\partial \xi_{3}}=0, & \xi_{ \pm} \in \Gamma_{ \pm}, \quad j=1,2, \ldots\end{cases}
\end{aligned}
$$

Hereinafter we employ the notation $\left(\xi_{1}^{ \pm}, \xi_{2}^{ \pm}, \xi_{3}^{ \pm}\right)=\xi_{ \pm}$, while $\Delta_{\xi_{ \pm}}$stands for the Laplace operator in variables $\xi_{ \pm}$.

We also denote $\rho_{ \pm}=\left|\xi_{ \pm}\right|, \mathbb{R}_{+}^{3, \pm}=\left\{\xi_{ \pm}: \xi_{3}^{ \pm}>0\right\}$. By $X_{j}^{ \pm}\left(\xi_{ \pm}\right)$and $X_{j}^{ \pm, i}\left(\xi_{ \pm}\right)$we denote homogeneous harmonic polynomials of degree $j$ satisfying the condition $\frac{\partial X_{j}^{ \pm}}{\partial \xi_{3}^{ \pm}}=\frac{\partial X_{j}^{ \pm, i}}{\partial \xi_{3}^{ \pm}}=0$ as $\xi_{3}^{ \pm}=0$. It follows from the definition that $X_{j}^{ \pm} \rho_{ \pm}^{-2 j-1}, X_{j}^{ \pm, i} \rho_{ \pm}^{-2 j-1}$ are harmonic functions satisfying condition $\frac{\partial X_{j}^{ \pm} \rho_{ \pm}^{-2 j-1}}{\partial \xi_{3}^{ \pm}}\left(\xi_{1}^{ \pm}, \xi_{2}^{ \pm}, 0\right)=\frac{\partial X_{j}^{ \pm, i} \rho_{ \pm}^{-2 j-1}}{\partial \xi_{3}^{ \pm}}\left(\xi_{1}^{ \pm}, \xi_{2}^{ \pm}, 0\right)=0$ as $\xi_{ \pm} \neq 0$. In [22, Lm. 3] the following statement was proven.

Lemma 1. For each function $\Phi \in C^{\infty}\left(\gamma_{ \pm}\right)$there exists a solution $v \in C^{\infty}\left(\overline{\mathbb{R}}_{+}^{3, \pm} \backslash \partial \gamma_{ \pm}\right) \cap$ $C\left(\overline{\mathbb{R}}_{+}^{3, \pm}\right)$ to the boundary value problem

$$
\begin{cases}\Delta v=0, & \xi_{3}^{ \pm}>0 \\ v=\Phi, & \xi_{ \pm} \in \gamma_{ \pm} \\ \frac{\partial v}{\partial \xi_{3}^{ \pm}}=0, & \xi_{ \pm} \in \Gamma_{ \pm}\end{cases}
$$


having the differentiable asymptotics

$$
v\left(\xi_{ \pm}\right)=\frac{C_{ \pm}(\Phi)}{\rho_{ \pm}}+\sum_{j=1}^{\infty} X_{j}^{ \pm}\left(\xi_{ \pm}\right) \rho_{ \pm}^{-1-2 j}, \quad \rho_{ \pm} \rightarrow \infty .
$$

We denote by $E\left(\xi_{ \pm} ; \gamma_{ \pm}\right)$the function satisfying Lemma 1 for $\Phi \equiv 1$. In terms of the notations of this lemma we have

$$
E\left(\xi_{ \pm} ; \gamma_{ \pm}\right)=\frac{C_{\gamma_{ \pm}}}{\rho_{ \pm}}+\sum_{j=1}^{\infty} X_{j}^{ \pm}\left(\xi_{ \pm}\right) \rho_{ \pm}^{-1-2 j}, \quad \rho_{ \pm} \rightarrow \infty
$$

where, we recall, $C_{\gamma_{ \pm}}>0$ are the capacities of disks $\gamma_{ \pm}$. We observe that if $\gamma_{ \pm}$is the unit circle, then

$$
E\left(\xi_{ \pm} ; \gamma_{ \pm}\right)=\frac{2}{\pi} \arctan \sqrt{\frac{2}{\rho_{ \pm}^{2}-1+\sqrt{\left(\rho_{ \pm}^{2}-1\right)^{2}+4\left(\xi_{3}^{ \pm}\right)^{2}}}}
$$

see, for instance, [14, Ch. 1, Sect. 4].

It follows from the definition of $E\left(\xi_{ \pm} ; \gamma_{ \pm}\right)$that the functions

$$
\left.v_{0}^{ \pm}\left(\xi_{ \pm}\right)= \pm \frac{\mathcal{B}_{0}}{C_{\gamma_{ \pm}}} E\left(\xi_{ \pm} ; \gamma_{ \pm}\right)\right) \pm\left(1-\frac{\mathcal{B}_{0}}{C_{\gamma_{ \pm}}}\right)
$$

solve boundary value problems (11) for each constant $\mathcal{B}_{0}$ and have the asymptotics

$$
v_{0}^{ \pm}\left(\xi_{ \pm}\right)= \pm\left(1-\frac{\mathcal{B}_{0}}{C_{\gamma_{ \pm}}}\right) \pm \frac{\mathcal{B}_{0}}{\rho_{ \pm}}+\sum_{j=1}^{\infty} \frac{X_{j}^{ \pm, 0}\left(\xi_{ \pm}\right)}{\rho_{ \pm}^{2 j+1}}, \quad \rho_{ \pm} \rightarrow \infty .
$$

Rewriting (13) in external variables $x_{ \pm}=\varepsilon \xi_{ \pm}$, we have

$$
v_{0}^{ \pm}\left(\frac{x^{ \pm}}{\varepsilon}\right)= \pm\left(1-\frac{\mathcal{B}_{0}}{C_{\gamma_{ \pm}}}\right) \pm \varepsilon \frac{\mathcal{B}_{0}}{r_{ \pm}}+\sum_{j=1}^{\infty} \varepsilon^{j} \frac{X_{j}^{ \pm, 0}\left(x_{ \pm}\right)}{r_{ \pm}^{2 j+1}}, \quad \varepsilon r_{ \pm} \rightarrow \infty .
$$

Hence, following the method of matching asymptotic expansions, we obtain that the external expansion should read as

$$
u(x, \varepsilon)=\sum_{k=0}^{\infty} \varepsilon^{k} u_{k}(x)
$$

where

$$
\begin{aligned}
u_{0}(x) & =\mathcal{A}_{0}^{ \pm}+o(1), \quad r_{ \pm} \rightarrow 0, \\
u_{1}(x) & = \pm \frac{\mathcal{B}_{0}}{r_{ \pm}}+O(1), \quad r_{ \pm} \rightarrow 0, \\
u_{j}(x) & =\frac{X_{j-1}^{ \pm, 0}\left(x_{ \pm}\right)}{r_{ \pm}^{2 j-1}}+O\left(r_{ \pm}^{-j+1}\right), \quad r_{ \pm} \rightarrow 0, j=2,3, \ldots \\
\mathcal{A}_{0}^{ \pm} & = \pm\left(1-\frac{\mathcal{B}_{0}}{C_{\gamma_{ \pm}}}\right) .
\end{aligned}
$$

Substituting expansion (14) into (8) and equating the coefficients at the like powers of $\varepsilon$, we obtain the following boundary value problems:

$$
\begin{cases}\Delta u_{k}=0, & x \in \Omega, \\ \frac{\partial u_{k}}{\partial \mathbf{n}}=0, & x \in \partial \Omega \backslash\left\{O_{+} \cup O_{-}\right\}, \quad k=0,1, \ldots\end{cases}
$$


By (4), the function

$$
u_{1}(x)=\mathcal{B}_{0}\left(G_{+}(x)-G_{-}(x)\right)+\mathcal{A}_{1}
$$

solves boundary value problem (19) and (16) for each constant $\mathcal{A}_{1}$.

It is obvious that if

$$
\mathcal{A}_{0}^{-}=\mathcal{A}_{0}^{+}:=\mathcal{A}_{0}
$$

then function

$$
u_{0}(x) \equiv \mathcal{A}_{0}
$$

satisfies (19) and (15).

Solving system of linear equations (18) and (21), we obtain that

$$
\mathcal{A}_{0}=\frac{C_{\gamma_{+}}-C_{\gamma_{-}}}{C_{\gamma_{+}}+C_{\gamma_{-}}}, \quad \mathcal{B}_{0}=\frac{2 C_{\gamma_{+}} C_{\gamma_{-}}}{C_{\gamma_{+}}+C_{\gamma_{-}}} .
$$

Thus, functions $u_{0}(x), v_{0}^{ \pm}\left(\xi_{ \pm}\right)$are well-defined thanks to identities (22), (12) and (23), while function $u_{1}(x)$ is defined by identity (20) up to an additive term $\mathcal{A}_{1}$.

In what follows by $Y_{j}^{ \pm}\left(\xi_{ \pm}\right)$and $Y_{j}^{ \pm, i}\left(\xi_{ \pm}\right)$we denote homogeneous harmonic polynomials of degree $j$ satisfying condition $\frac{\partial Y_{j}^{ \pm}}{\partial \xi_{3}^{ \pm}}=\frac{\partial Y_{j}^{ \pm, i}}{\partial \xi_{3}^{ \pm}}=0$ as $\xi_{3}^{ \pm}=0$. It follows from (20) that

$$
u_{1}(x)= \pm \frac{\mathcal{B}_{0}}{r_{ \pm}}+\mathcal{A}_{1} \mp \mathcal{B}_{0} G_{\mp}\left(O_{ \pm}\right)+\sum_{j=1}^{\infty} Y_{j}^{ \pm, 1}\left(x_{ \pm}\right), \quad r_{ \pm} \rightarrow 0 .
$$

Employing identities (22) and (24), we rewrite the asymptotics of the sum $u_{0}(x)+\varepsilon u_{1}(x)$ as $r_{ \pm} \rightarrow 0$ in terms of internal variables $\xi_{ \pm}$:

$$
u_{0}(x)+\varepsilon u_{1}(x)=\mathcal{A}_{0} \pm \frac{\mathcal{B}_{0}}{\rho_{ \pm}}+\varepsilon\left(\mathcal{A}_{1} \mp \mathcal{B}_{0} G_{\mp}\left(O_{ \pm}\right)\right)+\varepsilon \sum_{j=1}^{\infty} \varepsilon^{j} Y_{j}^{ \pm, 1}\left(\xi_{ \pm}\right) .
$$

It yields the asymptotics at infinity of functions $v_{k}^{ \pm}\left(\xi_{ \pm}\right)$:

$$
\begin{aligned}
& v_{0}^{ \pm}\left(\xi_{ \pm}\right)=\mathcal{A}_{0} \pm \frac{\mathcal{B}_{0}}{\rho_{ \pm}}+O\left(\rho_{ \pm}^{-2}\right), \quad \rho_{ \pm} \rightarrow \infty, \\
& v_{1}^{ \pm}\left(\xi_{ \pm}\right)=\mathcal{A}_{1} \mp \mathcal{B}_{0} G_{\mp}\left(O_{ \pm}\right)+O\left(\rho_{ \pm}^{-1}\right), \quad \rho_{ \pm} \rightarrow \infty, \\
& v_{k}^{ \pm}\left(\xi_{ \pm}\right)=Y_{k-1}^{ \pm, 1}\left(\xi_{ \pm}\right)+O\left(\rho_{ \pm}^{k-2}\right), \quad \rho_{ \pm} \rightarrow \infty, k=2,3, \ldots
\end{aligned}
$$

We note that functions $v_{0}^{ \pm}\left(\xi_{ \pm}\right)$defined in (12) have asymptotics (25) (see (18), (21)).

The definition of functions $E\left(\xi_{ \pm} ; \gamma_{ \pm}\right)$implies that for each constant $\mathcal{B}_{1}$, the functions

$$
v_{1}^{ \pm}\left(\xi_{ \pm}\right)= \pm \frac{\mathcal{B}_{1}}{C_{\gamma_{ \pm}}}\left(E\left(\xi_{ \pm} ; \gamma_{ \pm}\right)-1\right)
$$

solve boundary value problems (11) and have the asymptotics

$$
v_{1}^{ \pm}\left(\xi_{ \pm}\right)=\mp \frac{\mathcal{B}_{1}}{C_{\gamma_{ \pm}}} \pm \frac{\mathcal{B}_{1}}{\rho_{ \pm}}+\sum_{j=1}^{\infty} \frac{X_{j}^{ \pm, 1}\left(\xi_{ \pm}\right)}{\rho_{ \pm}^{2 j+1}}, \quad \rho_{ \pm} \rightarrow \infty .
$$

Comparing the latter identity with (26), we get the following system of linear equations for $\mathcal{A}_{1}$ and $\mathcal{B}_{1}$ :

$$
\mp \frac{\mathcal{B}_{1}}{C_{\gamma_{ \pm}}}=\mathcal{A}_{1} \mp \mathcal{B}_{0} G_{\mp}\left(O_{ \pm}\right)
$$


In view of (23) we obtain that, first,

$$
\begin{aligned}
\mathcal{A}_{1} & =\frac{2 C_{\gamma_{+}} C_{\gamma_{-}}}{\left(C_{\gamma_{+}}+C_{\gamma_{-}}\right)^{2}}\left(C_{\gamma_{+}} G_{-}\left(O_{+}\right)-C_{\gamma_{-}} G_{+}\left(O_{-}\right)\right), \\
\mathcal{B}_{1} & =2\left(\frac{C_{\gamma_{+}} C_{\gamma_{-}}}{C_{\gamma_{+}}+C_{\gamma_{-}}}\right)^{2}\left(G_{+}\left(O_{-}\right)+G_{-}\left(O_{+}\right)\right)
\end{aligned}
$$

and, second, asymptotics (28) specify asymptotics (26).

By means of identities (13) and (28) , we rewrite the asymptotics of the sums $v_{0}^{ \pm}\left(\xi_{ \pm}\right)+\varepsilon v_{1}^{ \pm}\left(\xi_{ \pm}\right)$ as $\rho_{ \pm} \rightarrow \infty$ in external variables $x_{ \pm}$:

$$
\begin{aligned}
v_{0}^{ \pm}\left(\xi_{ \pm}\right)+\varepsilon v_{1}^{ \pm}\left(\xi_{ \pm}\right)= & \mathcal{A}_{0}+\varepsilon\left( \pm \frac{\mathcal{B}_{0}}{r_{ \pm}}+\mathcal{A}_{1} \mp \mathcal{B}_{0} G_{\mp}\left(O_{ \pm}\right)\right)+\varepsilon^{2}\left(\frac{X_{1}^{ \pm, 0}\left(x_{ \pm}\right)}{r_{ \pm}^{3}} \pm \frac{\mathcal{B}_{1}^{ \pm}}{r_{ \pm}}\right)+ \\
& +\sum_{j=3}^{\infty} \varepsilon^{j}\left(\frac{X_{j-1}^{ \pm, 0}\left(x_{ \pm}\right)}{r_{ \pm}^{2 j-1}}+\frac{X_{j-2}^{ \pm, 1}\left(x_{ \pm}\right)}{r_{ \pm}^{2 j-3}}\right) .
\end{aligned}
$$

Following the method of matching asymptotic expansions, we specify asymptotics (15), (16), (17):

$$
\begin{aligned}
& u_{0}(x)=\mathcal{A}_{0}+o(1), \quad u_{1}(x)= \pm \frac{\mathcal{B}_{0}}{r_{ \pm}}+\mathcal{A}_{1} \mp \mathcal{B}_{0} G_{\mp}\left(O_{ \pm}\right)+O\left(r_{ \pm}\right), \quad r_{ \pm} \rightarrow 0, \\
& u_{2}(x)=\frac{X_{1}^{ \pm, 0}\left(x_{ \pm}\right)}{r_{ \pm}^{3}} \pm \frac{\mathcal{B}_{1}}{r_{ \pm}}+O(1), \quad r_{ \pm} \rightarrow 0, \\
& u_{j}(x)=\frac{X_{j-1}^{ \pm, 0}\left(x_{ \pm}\right)}{r_{ \pm}^{2 j-1}}+\frac{X_{j-2}^{ \pm, 1}\left(x_{ \pm}\right)}{r_{ \pm}^{2 j-3}}+O\left(r_{ \pm}^{-j+2}\right), \quad r_{ \pm} \rightarrow 0, j=3,4, \ldots
\end{aligned}
$$

Functions $u_{0}(x)$ and $u_{1}(x)$ defined by identities (22) and (20) satisfy (30) (cf. (24)).

Lemma 2 in [22] implies the following statement.

Lemma 2. Let $n \geqslant 1$. Then for each $X_{n}^{ \pm}\left(x_{ \pm}\right)$there exist functions $U_{ \pm}(x) \in C^{\infty}\left(\bar{\Omega} \backslash O_{ \pm}\right)$ solving boundary value problems

$$
\left\{\begin{array}{l}
\Delta U_{ \pm}=0, \quad x \in \Omega \\
\frac{\partial U_{ \pm}}{\partial \mathbf{n}}=0, \quad x \in \partial \Omega \backslash O_{ \pm}
\end{array}\right.
$$

and having differentiable asymptotics

$$
U_{ \pm}(x)=\frac{X_{n}^{ \pm}\left(x_{ \pm}\right)}{r_{ \pm}^{2 n+1}}+\sum_{j=1}^{\infty} Y_{j}^{ \pm}\left(x_{ \pm}\right), \quad r_{ \pm} \rightarrow 0 .
$$

By (41) and Lemma 2, there exists a solution $u_{2}(x) \in C^{\infty}\left(\bar{\Omega} \backslash\left\{O_{+} \cup O_{-}\right\}\right)$to boundary value problem (19), (31), which can be represented as

$$
u_{2}(x)=\frac{X_{1}^{+, 0}\left(x_{+}\right)}{r_{+}^{3}}+\frac{X_{1}^{-, 0}\left(x_{-}\right)}{r_{-}^{3}}+\frac{\mathcal{B}_{1}}{r_{+}}-\frac{\mathcal{B}_{1}}{r_{-}}+\widetilde{u}_{2}(x)+\mathcal{A}_{2},
$$

where $\widetilde{u}_{2} \in C^{\infty}(\bar{\Omega}), \int_{\Omega} \widetilde{u}_{2}(x) d x=0$, and $\mathcal{A}_{2}$ is an arbitrary constant. 
In particular, it follows from (22), (20), (32), (12), (27), (23) and (29) that

$$
\begin{aligned}
u_{0}(x) \equiv & \frac{C_{\gamma_{+}}-C_{\gamma_{-}}}{C_{\gamma_{+}}+C_{\gamma_{-}}}, \\
u_{1}(x)= & \frac{2 C_{\gamma_{+}} C_{\gamma_{-}}}{C_{\gamma_{+}}+C_{\gamma_{-}}}\left(G_{+}(x)-G_{-}(x)+\frac{1}{C_{\gamma_{+}}+C_{\gamma_{-}}}\left(C_{\gamma_{+}} G_{-}\left(O_{+}\right)-C_{\gamma_{-}} G_{+}\left(O_{-}\right)\right)\right), \\
u_{2}(x)= & \frac{X_{1}^{+, 0}\left(x_{+}\right)}{r_{+}^{3}}+\frac{X_{1}^{-}, 0\left(x_{-}\right)}{r_{-}^{3}}+ \\
& +2\left(\frac{C_{\gamma_{+}} C_{\gamma_{-}}}{C_{\gamma_{+}}+C_{\gamma_{-}}}\right)^{2}\left(G_{+}\left(O_{-}\right)+G_{-}\left(O_{+}\right)\right)\left(\frac{1}{r_{+}}-\frac{1}{r_{-}}\right)+\widetilde{u}_{2}(x)+\mathcal{A}_{2},
\end{aligned}
$$

where $\widetilde{u}_{2} \in C^{\infty}(\bar{\Omega})$, and $\mathcal{A}_{2}$ is an arbitrary constant,

$$
\begin{aligned}
& v_{0}^{ \pm}\left(\xi_{ \pm}\right)= \pm\left(1+\frac{2 C_{\gamma_{\mp}}}{C_{\gamma_{+}}+C_{\gamma_{-}}}\left(E\left(\xi_{ \pm} ; \gamma_{ \pm}\right)-1\right)\right) \\
& v_{1}^{ \pm}\left(\xi_{ \pm}\right)= \pm 2 \frac{C_{\gamma_{\mp}} C_{\gamma_{ \pm}}^{2}}{\left(C_{\gamma_{+}}+C_{\gamma_{-}}\right)^{2}}\left(G_{+}\left(O_{-}\right)+G_{-}\left(O_{+}\right)\right)\left(E\left(\xi_{ \pm} ; \gamma_{ \pm}\right)-1\right) .
\end{aligned}
$$

Thus, we have constructed leading terms $u_{0}(x), u_{1}(x), u_{2}(x)$ (up to an additive constant $\mathcal{A}_{2}$ ), $v_{0}^{ \pm}\left(\xi_{ \pm}\right), v_{1}^{ \pm}\left(\xi_{ \pm}\right)$of formal expansions (14) and (9). The construction of complete asymptotic expansions (14) and (9) and their rigorous justification will be provided in the next two sections.

\section{Formal CONSTRUCTION OF COMPLETE ASYMPTOTICS FOR SOLUTION TO BOUNDARY VALUE PROBLEM (8)}

This section is devoted to the proof of the following statement.

Theorem 1. There exist series (14), (9) such that

1) functions $u_{k}(x) \in C^{\infty}\left(\bar{\Omega} \backslash\left\{O_{+} \cup O_{-}\right\}\right)$solve boundary value problems (19);

2) functions $u_{0}(x), u_{1}(x), u_{2}(x)$ satisfy identities (33), where $\widetilde{u}_{2} \in C^{\infty}(\bar{\Omega})$, and $\mathcal{A}_{2}$ is some constant;

3) as $k \geqslant 1$, functions $u_{k}(x)$ have the following asymptotics:

$$
u_{k}(x)=\sum_{q=1}^{k-1} \frac{X_{q}^{ \pm, k-q-1}\left(x_{ \pm}\right)}{r_{ \pm}^{2 q+1}} \pm \frac{\mathcal{B}_{k-1}}{r_{ \pm}}+\mathcal{A}_{k}^{ \pm}+\sum_{j=1}^{\infty} Z_{j}^{ \pm, k}\left(x_{ \pm}\right), \quad x_{ \pm} \rightarrow 0
$$

4) functions $v_{k}^{ \pm}\left(\xi_{ \pm}\right) \in C^{\infty}\left(\overline{\mathbb{R}}_{+}^{3, \pm} \backslash \partial \gamma_{ \pm}\right) \cap C\left(\overline{\mathbb{R}}_{+}^{3, \pm}\right)$ solve boundary value problems (10), (11);

5) functions $v_{0}^{ \pm}\left(\xi_{ \pm}\right)$and $v_{1}^{ \pm}\left(\xi_{ \pm}\right)$satisfy identities (34);

6) functions $v_{k}^{ \pm}\left(\xi_{ \pm}\right)$have the following asymptotics:

$$
v_{k}^{ \pm}\left(\xi_{ \pm}\right)=\sum_{q=1}^{k-1} Z_{q}^{ \pm, k-q}\left(\xi_{ \pm}\right)+\mathcal{A}_{k}^{ \pm} \pm \frac{\mathcal{B}_{k}}{\rho_{ \pm}}+\sum_{j=1}^{\infty} \frac{X_{j}^{ \pm, k}\left(\xi_{ \pm}\right)}{\rho_{ \pm}^{2 j+1}}, \quad \rho_{ \pm} \rightarrow \infty .
$$

Proof. Formulae (36) for $v_{0}^{ \pm}$are implied by (13) as $\pm\left(1-\frac{\mathcal{B}_{0}}{C_{\gamma_{ \pm}}}\right):=\mathcal{A}_{0}^{ \pm}$, while for $v_{1}^{ \pm}$they are implied by (28) as $\mp \frac{\mathcal{B}_{1}}{C_{\gamma_{+}}}:=\widetilde{\mathcal{A}}_{1}^{ \pm}$. Identity (35) for $u_{1}$ coincides with (24).

It follows from (32) that

$$
u_{2}(x)=\frac{X_{1}^{ \pm, 0}\left(x_{ \pm}\right)}{r_{ \pm}^{3}} \pm \frac{\mathcal{B}_{1}}{r_{ \pm}}+\widetilde{\mathcal{A}}_{2}^{ \pm}+\mathcal{A}_{2}+\sum_{j=1}^{\infty} Z_{j}^{ \pm, 2}\left(x_{ \pm}\right), \quad x_{ \pm} \rightarrow 0,
$$

where $\widetilde{\mathcal{A}}_{2}^{ \pm}$are fixed numbers, and $\mathcal{A}_{2}$ is an arbitrary constant. 
By Lemma 1, there exist solutions $\widetilde{v}_{2}^{ \pm} \in C^{\infty}\left(\overline{\mathbb{R}}_{+}^{3, \pm} \backslash \partial \gamma_{ \pm}\right) \cap C\left(\mathbb{R}_{+}^{3, \pm}\right)$ to boundary value problem (11) with the asymptotics

$$
\widetilde{v}_{2}^{ \pm}\left(\xi_{ \pm}\right)=Z_{1}^{ \pm, 1}\left(\xi_{ \pm}\right) \pm \frac{\widetilde{\mathcal{B}}_{2}^{ \pm}}{\rho_{ \pm}}+O\left(\rho_{ \pm}^{-2}\right), \quad \rho_{ \pm} \rightarrow \infty
$$

where $\widetilde{\mathcal{B}}_{2}^{ \pm}$are fixed numbers. Then for each numbers $\mathcal{B}_{2}^{ \pm}$, the functions

$$
v_{2}^{ \pm}\left(\xi_{ \pm}\right)=\widetilde{v}_{2}^{ \pm}\left(\xi_{ \pm}\right) \pm \frac{\mathcal{B}_{2}^{ \pm}}{C_{\gamma_{ \pm}}}\left(E\left(\xi_{ \pm} ; \gamma_{ \pm}\right)-1\right) \in C^{\infty}\left(\overline{\mathbb{R}}_{+}^{3, \pm} \backslash \partial \gamma_{ \pm}\right) \cap C\left(\mathbb{R}_{+}^{3, \pm}\right)
$$

are solutions to boundary value problems (11) with the following asymptotics

$$
v_{2}^{ \pm}\left(\xi_{ \pm}\right)=Z_{1}^{ \pm, 1}\left(\xi_{ \pm}\right) \mp \frac{\mathcal{B}_{2}^{ \pm}}{C_{\gamma_{ \pm}}} \pm \frac{\widetilde{\mathcal{B}}_{2}^{ \pm}+\mathcal{B}_{2}^{ \pm}}{\rho_{ \pm}}+O\left(\rho_{ \pm}^{-2}\right), \quad \rho_{ \pm} \rightarrow \infty .
$$

Comparing the latter identity with required identity (36) for $k=2$, we obtain a linear equation for $\mathcal{B}_{2}^{+}$and $\mathcal{B}_{2}^{-}$:

$$
\widetilde{\mathcal{B}}_{2}^{+}+\mathcal{B}_{2}^{+}=\widetilde{\mathcal{B}}_{2}^{-}+\mathcal{B}_{2}^{-}:=\mathcal{B}_{2} .
$$

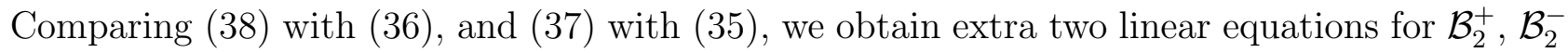
and $\mathcal{A}_{2}$ :

$$
\mp \frac{\mathcal{B}_{2}^{ \pm}}{C_{\gamma_{ \pm}}}=\widetilde{\mathcal{A}}_{2}^{ \pm}+\mathcal{A}_{2}:=\mathcal{A}_{2}^{ \pm} .
$$

Since the determinant of system of equations (39), (40) is equal to $\frac{1}{C_{\gamma_{+}}}+\frac{1}{C_{\gamma_{-}}}$, it is uniquely solvable. Calculating $\mathcal{B}_{2}^{+}, \mathcal{B}_{2}^{-}$and $\mathcal{A}_{2}$, we find $\mathcal{B}_{2}, \mathcal{A}_{2}^{ \pm}$by (39), (40) and therefore, we finally determine $v_{2}^{ \pm}\left(\xi_{ \pm}\right)$and $u_{2}(x)$ achieving at that identities (35) and (36) for $k=2$.

Moreover, by Lemma 2 there exists solution $u_{3}(x) \in C^{\infty}\left(\bar{\Omega} \backslash\left\{O_{+} \cup O_{-}\right\}\right)$to boundary value problem (19) with the asymptotics

$$
u_{3}(x)=\frac{X_{2}^{ \pm, 0}\left(x_{ \pm}\right)}{r_{ \pm}^{5}}+\frac{X_{1}^{ \pm, 1}\left(x_{ \pm}\right)}{r_{ \pm}^{3}} \pm \frac{\mathcal{B}_{2}}{r_{ \pm}}+\widetilde{\mathcal{A}}_{3}^{ \pm}+\mathcal{A}_{3}+\sum_{j=1}^{\infty} Z_{j}^{ \pm, 3}\left(x_{ \pm}\right), \quad x_{ \pm} \rightarrow 0,
$$

where $\widetilde{\mathcal{A}}_{3}^{ \pm}$are fixed numbers and $\mathcal{A}_{3}$ is an arbitrary constant. That is, we have obtained an analogue of identity (37) for the next step.

Repeating the above described procedure, we find successively $\mathcal{B}_{3}, \mathcal{A}_{3}^{ \pm}, \mathcal{A}_{3}$, determine finally $v_{3}^{ \pm}\left(\xi_{ \pm}\right)$and $u_{3}(x)$ and achieve identities (35) and (36) for $k=3$, while by Lemma 2 we obtain the existence of solution $u_{4}(x) \in C^{\infty}\left(\bar{\Omega} \backslash\left\{O_{+} \cup O_{-}\right\}\right)$to boundary value problem (19) with the asymptotics

$$
u_{4}(x)=\frac{X_{3}^{ \pm, 0}\left(x_{ \pm}\right)}{r_{ \pm}^{7}}+\frac{X_{2}^{ \pm, 1}\left(x_{ \pm}\right)}{r_{ \pm}^{5}}+\frac{X_{1}^{ \pm, 2}\left(x_{ \pm}\right)}{r_{ \pm}^{3}} \pm \frac{\mathcal{B}_{3}}{r_{ \pm}}+\widetilde{\mathcal{A}}_{4}^{ \pm}+\mathcal{A}_{4}+\sum_{j=1}^{\infty} Z_{j}^{ \pm, 4}\left(x_{ \pm}\right), \quad x_{ \pm} \rightarrow 0,
$$

where $\widetilde{\mathcal{A}}_{4}^{ \pm}$are fixed numbers and $\mathcal{A}_{4}$ is an arbitrary constant, and so forth. The proof is complete.

\section{JUSTIFICATION OF CONSTRUCTED ASYMPtOTICS FOR SOLUTION TO BOUNDARY VALUE PROBLEM (8)}

We denote by $B_{ \pm}(t)$ the ball of radius $t$ centered at point $O_{ \pm}$. This section is devoted to the proof of the following section based on the approach employed in justifying the asymptotics for the eigenvalues of boundary value problems with a change of type of boundary condition on a small part of a boundary [23 26]. 
Theorem 2. Suppose that functions $u_{k}(x)$ and $v_{k}^{ \pm}\left(\xi_{ \pm}\right)$satisfy the hypothesis of Theorem 1. Then the solution to boundary value problem (8) has asymptotics (14) in $\Omega \backslash\left(B_{+}(\sqrt{\varepsilon}) \cup B_{-}(\sqrt{\varepsilon})\right)$ and asymptotics (9) in $\Omega \cap B_{ \pm}(\sqrt{\varepsilon})$ in the sense of $W_{2}^{1}$-norm.

Proof. We denote by $\widehat{u}_{N}(x, \varepsilon)$ and $\widehat{v}_{N}^{ \pm}(x, \varepsilon)$ partial sums of series (14), (9), respectively, up to the powers $\varepsilon^{N}$. Statements 3) and 6) of Theorem 1 imply the following differentiable identity

$$
\widehat{u}_{N}(x, \varepsilon)-\widehat{v}_{N}^{ \pm}(x, \varepsilon)=O\left(\varepsilon r_{ \pm}^{N}+\varepsilon^{N} r_{ \pm}+\left(\frac{\varepsilon}{r_{ \pm}}\right)^{N+1}\right), \quad r_{ \pm} \rightarrow 0, \quad \frac{r_{ \pm}}{\varepsilon} \rightarrow \infty .
$$

Let $\chi(t)$ be an infinitely differentiable cut-off function vanishing as $t<1$ and being equal to one as $t>2$. We denote

$$
\begin{aligned}
\widetilde{u}_{N, s}(x, \varepsilon)= & \widehat{u}_{N}(x, \varepsilon) \chi\left(\frac{s r_{+}}{\sqrt{\varepsilon}}\right) \chi\left(\frac{s r_{-}}{\sqrt{\varepsilon}}\right)+ \\
& +\widehat{v}_{N}^{+}(x, \varepsilon)\left(1-\chi\left(\frac{s r_{+}}{\sqrt{\varepsilon}}\right)\right)+\widehat{v}_{N}^{-}(x, \varepsilon)\left(1-\chi\left(\frac{s r_{-}}{\sqrt{\varepsilon}}\right)\right) .
\end{aligned}
$$

Then function $\widetilde{u}_{N, s}(x, \varepsilon)$ solves boundary value problem

$$
\begin{cases}\Delta \widetilde{u}_{N, s}=f_{N, s}, & x \in \Omega, \\ \frac{\partial \widetilde{u}_{N, s}}{\partial \mathbf{n}}=0, & x \in \partial \Omega \backslash\left\{\gamma_{+}^{\varepsilon} \cup \gamma_{-}^{\varepsilon}\right\}, \\ \widetilde{u}_{N, s}= \pm 1, & x \in \gamma_{ \pm}^{\varepsilon},\end{cases}
$$

where

$$
\begin{aligned}
f_{N, s}(x, \varepsilon)= & \left(\widehat{u}_{N}(x, \varepsilon)-\widehat{v}_{N}^{ \pm}(x, \varepsilon)\right) \Delta \chi\left(\frac{s r_{+}}{\sqrt{\varepsilon}}\right)+\left(\widehat{u}_{N}(x, \varepsilon)-\widehat{v}_{N}^{ \pm}(x, \varepsilon)\right) \Delta \chi\left(\frac{s r_{-}}{\sqrt{\varepsilon}}\right) \\
& +\nabla\left(\widehat{u}_{N}(x, \varepsilon)-\widehat{v}_{N}^{ \pm}(x, \varepsilon)\right) \nabla \chi\left(\frac{s r_{+}}{\sqrt{\varepsilon}}\right)+\left(\widehat{u}_{N}(x, \varepsilon)-\widehat{v}_{N}^{ \pm}(x, \varepsilon)\right) \nabla \chi\left(\frac{s r_{-}}{\sqrt{\varepsilon}}\right) .
\end{aligned}
$$

By (41) it implies that

$$
\left\|f_{N, s}\right\|_{L_{2}(\Omega)}=O\left(\varepsilon^{\frac{N}{2}}\right)
$$

Denote

$$
U_{N, s}(x, \varepsilon)=\widetilde{u}_{N, s}(x, \varepsilon)-u(x, \varepsilon) .
$$

By (8) and (43) we obtain that function $U_{N, s}(x, \varepsilon)$ is a solution to the boundary value problem

$$
\begin{cases}\Delta U_{N, s}=f_{N, s}, & x \in \Omega, \\ \frac{\partial U_{N, s}}{\partial \mathbf{n}}=0, & x \in \partial \Omega \backslash\left\{\gamma_{+}^{\varepsilon} \cup \gamma_{-}^{\varepsilon}\right\}, \\ U_{N, s}=0, & x \in \gamma_{ \pm}^{\varepsilon} .\end{cases}
$$

We multiply equation in (46) by $U_{N, s}(x, \varepsilon)$ and integrate the obtained identity over $\Omega$. Integrating by parts in the left hand side of the latter identity, by (44) we have

$$
\left\|\nabla U_{N, s}\right\|_{L_{2}(\Omega)}=O\left(\varepsilon^{\frac{N}{2}}\right) \text {. }
$$

It was shown in [27] that the minimal eigenvalue of the boundary value problem

$$
\begin{cases}-\Delta \psi_{\varepsilon}=\lambda_{\varepsilon} \psi_{\varepsilon}, & x \in \Omega, \\ \frac{\partial \psi_{\varepsilon}}{\partial \mathbf{n}}=0, & x \in \partial \Omega \backslash \gamma_{+}^{\varepsilon}, \\ \psi_{\varepsilon}=0, & x \in \gamma_{+}^{\varepsilon},\end{cases}
$$


has the asymptotics $\lambda_{\varepsilon}=\varepsilon 2 \pi C_{\gamma_{+}}|\Omega|+O\left(\varepsilon^{2}\right)$. Hence, variational properties of the eigenvalues of boundary value problems imply (see, for instance, [16, Ch. IV, Sect. 1, Subsect. 4]) that for the functions in $W_{2}^{1}(\Omega)$ vanishing on $\gamma_{+}$the estimate

$$
\|w\|_{L_{2}(\Omega)}^{2} \leqslant \frac{1}{\varepsilon \pi C_{\gamma_{+}}|\Omega|}\|\nabla w\|_{L_{2}(\Omega)}^{2}
$$

holds true for sufficiently small $\varepsilon$. Together with (47) and (45) it yields the identity

$$
\left\|\widetilde{u}_{N, s}-u\right\|_{W_{2}^{1}(\Omega)}=O\left(\varepsilon^{\frac{N-1}{2}}\right) .
$$

Finally, this identity, definition (42) of function $\widetilde{u}_{N, s}(x, \varepsilon)$ and the arbitrary choice of $N$ and $s$ complete the proof of the theorem.

\section{Proof of formulae (2), (3)}

Let $H$ be an arbitrary cross-section of domain $\Omega$ not containing points $O_{ \pm}$, and $H_{\mu}$ be the $\mu$-neighborhood of $H$ for sufficiently small $\mu$. Then the solution to boundar value problem (46) satisfies estimate [16, Ch. IV, Sect. 2]

$$
\left\|U_{N, s}\right\|_{W_{2}^{2}\left(H_{\mu}\right)} \leqslant C\left\|f_{N, s}\right\|_{L_{2}(\Omega)}
$$

Since

$$
\|w\|_{L^{2}(H)} \leqslant C_{1}\|w\|_{W_{2}^{1}\left(H_{\mu}\right)},
$$

[16. Ch. III, Sect. 5], it follows from (48), (44) and the inequality $\|w\|_{L_{1}(H)} \leqslant|H|\|w\|_{L_{2}(H)}$ that

$$
\int_{H}\left|\frac{\partial U_{N, s}}{\partial \mathbf{n}_{H}}\right| d S=O\left(\varepsilon^{\frac{N}{2}}\right)
$$

By definitions (45) and (42) of functions $U_{N, s}(x, \varepsilon)$ and $\widetilde{u}_{N, s}(x, \varepsilon)$ and arbitrary choice of $N$ it yields that

$$
\int_{H} \frac{\partial u}{\partial \mathbf{n}_{H}} d S=\int_{H} \frac{\partial \widehat{u}_{N}}{\partial \mathbf{n}_{H}} d S+O\left(\varepsilon^{N+1}\right) .
$$

Substituting (49) into (6), in view of identities (22) and (피) and [22, Lm. 2] we obtain that

$$
I=\sigma\left|\sum_{k=1}^{N} \varepsilon^{k} \mathcal{B}_{k-1} \int_{H} \frac{\partial}{\partial \mathbf{n}_{H}}\left(\frac{1}{r_{+}}-\frac{1}{r_{-}}\right) d S\right|+O\left(\varepsilon^{N+1}\right)=2 \pi \sigma\left|\sum_{k=1}^{N} \varepsilon^{k} \mathcal{B}_{k-1}\right|+O\left(\varepsilon^{N+1}\right) .
$$

Since $\mathcal{B}_{0}>0$ and $\mathcal{B}_{1}>0$ by (23) and (29), substituting (150) and $\Delta U=2$ into (5), by arbitrary choice of $N$ we arrive at formula (2) for $R(\varepsilon)$, where

$$
R_{-1}=\frac{1}{\pi \sigma \mathcal{B}_{0}}, \quad R_{0}=-\frac{\mathcal{B}_{1}}{\pi \sigma \mathcal{B}_{0}^{2}}
$$

And finally, the latter identities and values (23), (29) of constants $\mathcal{B}_{0}, \mathcal{B}_{1}$ we obtain formulae (3) for $R_{-1}$ and $R_{0}$. 


\section{BIBLIOGRAPHY}

1. R. Holm. Über Kontaktwiderstände, besonders bei Kohlekontakten // Zeit. Tech. Phys. 3:9, 290-294 (1922).

2. R. Holm, R. Störmer. Eine Kontrolle des metallischen Charakters von gereinigten Platinkontakten // Wissenschaftliche Veröffentlichungen aus dem Siemens-Konzern. 9:2, 323-330 (1930).

3. R. Holm. Electric constacts. Springer-Verlag, Berlin (1958).

4. O.M. Pavleino, V.A. Pavlov, M.A. Pavleino. Effect of the spreading of the contact spot on the pulsed heating of electrodes // Elektron. Obrabotka Mater. 47:4, 142-149 (2011). [Surf. Eng. Appl. Electrochem. 47:4, 142-149 (2011).]

5. I.V. Plokhov. Model of dynamics of current transfer through sliding contact // Elektrotechnika. 2, 28-33 (2005). (in Russian).

6. V.V. Filippov, N.N. Polyakov. Potential distribution in anisotropic conductor cristalls and films in measuring electric conductivity and Hall coefficient // Bull. Univ. Chernozem'e. 24:2, 6-10 (2011). (in Russian).

7. A.A. Ershov. Asymptotics of the solution to the Neumann problem with a delta-function-like boundary function // Zhurn. Vychisl. Matem. Matem. Fiz. 50:3, 479-485 (2010). [Comp. Math. Math. Phys. 50:3, 457-463 (2010).]

8. A.A. Ershov. Asymptotics of the solution of Laplace's equation with mixed boundary conditions // Zhurn. Vychisl. Matem. Matem. Fiz. 51:7, 1064-1080 (2011). [Comp. Math. Math. Phys. 51:7, 994-1010 (2011).]

9. A.A. Ershov. On measurement of electrical conductivity // Zhurn. Vychisl. Matem. Matem. Fiz. 53:6, 1004-1007 (2013). [Comp. Math. Math. Phys. 53:6, 823-826 (2013).]

10. G. Pólya, G. Szegö. Isoperimetric inequalities in mathematical physics. Princeton Univ. Press, Princeton (1951).

11. N.S. Landkof. Foundations of modern potential theory. Nauka, Moscow (1966). [Springer, Berlin (1972).]

12. V.I. Smirnov. A course of higher mathematics. V. IV. Moscow, Nauka (1981). [Pergamon Press, Oxford (1964).]

13. A.M. Il'in. Equations of mathematical physics. Nauka, Moscow (2009). (in Russian).

14. L.D. Landau, E.M. Lifschitz. Theoretical physics. V. VIII. Electrodynamics of continuous media. Fizmatlit, Moscow (2005). [Butterworth-Heinemann, Oxford (1984).]

15. S. Zaremba. Sur un problème mixte relatif a l'équation de Laplace // Krakau Anz. (A). 313-344 (1910).

16. V.P. Mikhajlov. Partial differential equations. Nauka, Moscow (1976). [Mir Publishers, Moscow (1978).]

17. R.R. Gadyl'shin. Asymptotic properties of an eigenvalue of a problem for a singularly perturbed self-adjoint elliptic equation with a small parameter in the boundary conditions // Differ. Uravn. 22:4, 640-652 (1986). [Differ. Equat. 22:4, 474-483 (1986).]

18. A.M. Il'in. Matching of asymptotic expansions of solutions of boundary value problems. Nauka, Moscow (1989). [Transl. Math. Monographs. AMS, Providence, RI (1992).]

19. V. Maz'ya, S. Nazarov, B. Plamenevsky. Asymptotic theory of elliptic boundary value problems in singularly perturbed domains V. 1,2. Birkhaeuser Verlag, Basel (2000).

20. R.R. Gadyl'shin. Concordance method of asymptotic expansions in a singularly-perturbed boundary-value problem for the Laplace operator // Sovrem. Mat. Prilozh. Asimptot. Metody Funkts. Anal. 3-32 (2003). [J. Math. Sci. 125:5, 579-609 (2005).]

21. A.R. Bikmetov, R.R. Gadyl'shin. Perturbation of an elliptic operator by a narrow potential in an n-dimensional domain // Ufimskij Matem. Zhurn. 4:2, 28-64 (2012). [Ufa Math. J. 4:2, 28-64 (2012).]

22. A.A. Ershov. Mixed problem for a harmonic function // Zhurn. Vychisl. Matem. Matem. Fiz. 53:7, 1094-1106 (2013). [Comp. Math. Math. Phys. 53:7, 908-919 (2013).] 
23. R.R. Gadyl'shin. Ramification of a multiple eigenvalue of the Dirichlet problem for the Laplacian under singular perturbation of the boundary condition // Matem. Zametki. 52:4, 42-55 (1992). [Math. Notes. 52:4, 1020-1029 (1992).]

24. R.R. Gadyl'shin. Splitting a multiple eigenvalue in the boundary value problem for a membrane clamped on a small part of the boundary // Sibir. Matem. Zhurn. [Siber. Math. J. 34:3, 433-450 (1993).]

25. R.R. Gadyl'shin, E.A. Shishkina. On Friedrichs inequalities for a disk // Trudy IMM UrO RAN. 18:2, 48-61 (2012). [Proc. Steklov Inst. Math. Suppl. 1. 281, 44-58 (2013).]

26. R.R. Gadyl'shin, S.V. Repjevskij, E.A. Shishkina. On an eigenvalue for the Laplace operator in a disk with Dirichlet boundary condition on a small part of the boundary in a critical case // Trudy IMM UrO RAN. 21:1, 56-70 (2015). [Proc. Steklov Inst. Math. Suppl. 1. 284, (2016), to appear.]

27. R.R. Gadyl'shin. On the perturbation of the Laplacian spectrum when the boundary condition type changes on a small part of the boundary // Zhurn. Vychisl. Matem. Matem. Fiz. 36:7, 77-88 (1996). [Comp. Math. Math. Phys. 36:7, 889-898 (1996).]

Rustem Rashitovich Gadyl'shin, Akhmulla Bashkir State Pedagogical University,

October rev. st. 3a,

450000, Ufa, Russia

E-mail: Gadylshin@yandex.ru

Alexander Anatol'evich Ershov, Chelyabnisk State University,

Br. Kashiriny str. 129,

454001, Chelyabinsk, Russia

E-mail: Ale10919@yandex.ru

Sergei Vladimirovich Rep'evskij, Chelyabnisk State University,

Br. Kashiriny str. 129,

454001, Chelyabinsk, Russia

E-mail: Repyevsky@gmail.com 